\title{
THE STRUCTURE AND PROPERTIES OF HARDENED STAINLESS STEELS WITH A SHAPE MEMORY EFFECT
}

\author{
S. V. Afanasiev, E. G. Volkova*, V. V. Sagaradze \\ M.N. Miheev Institute of Metal Physics of Ural Branch of Russian Academy of Sciences, 18 S. Kovalevskoy st., \\ Ekaterinburg, Russian Federation \\ *Corresponding author. E-mail: volkova@imp.uran.ru; address for correspondence: 18, ul. S. Kovalevskoy, 620990, \\ Ekaterinburg, Russian Federation. Tel.: +7 343 3783527; fax: +7 3433745244
}

A possibility of obtaining the shape memory effect has been investigated as a result of $\gamma \rightarrow \varepsilon \rightarrow \gamma$ transformations in stainless steels hardened by $\mathrm{VC}$ carbides. Age hardening conditions at 650 and $720{ }^{\circ} \mathrm{C}$ for stainless steels containing (in mass \%) $0.20-0.35 \mathrm{C}, 1 \mathrm{~V}, 3-4 \mathrm{Si}, 9$ and $13-14 \mathrm{Cr}$ are presented. The values of reversible strain $e$ determined after heating to $400{ }^{\circ} \mathrm{C}$ in specimens preliminarily deformed by $3.5-4 \%$ vary from 0.15 to $2.7 \%$ depending on the composition of the steels and the conditions of stabilizing and destabilizing aging.

Keywords: shape memory effect, stainless steel, VC carbide, martensite transformation, $\varepsilon$-phase, electron microscopy.

DOI: $10.17804 / 2410-9908.2015 .6 .019-026$

\section{References}

1. $\quad$ Sagaradze V.V., Belozerov Ye.V., Pecherkina N.L., Mukhin M.L., Zaynutdinov Yu.R. The shape memory effect in high-strength precipitation-hardening austenitic steels. Materials Science and Engineering: A, 2006, vol. 438-440, pp. 812-815. DOI:10.1016/j.msea.2006.01.113.

2. Sagaradze V.V., Belozerov E.V., Mukhin M.L., Zainutdinov Yu.R., Pecherkina N.L., Zavalishin V.A. A new approach to creation of high-strength austenitic steels with a controlled shape-memory effect. The Physics of Metals and Metallography, 2006, vol. 101, iss. 5, pp. 506-512. DOI: 10.1134/S0031918X06050127.

3. $\quad$ Sagaradze V.V., Mukhin M.L., Belozerov E.V., Zainutdinov Yu.R., Pecherkina N.L., Filippov Yu.I. Controlled shape memory effect in high strength $\mathrm{Mn}$ and Cr-Mn steels. Materials Science and Engineering: A, 2008, vol. 481-482, pp. 742-746. DOI:10.1016/j.msea.2007.02.155.

4. Sagaradze V.V., Kositsyna I.I., Mukhin M.L., Belozerov E.V., Zaynutdinov Yu.R. Highstrength precipitation-hardening austenitic Fe-Mn-V-Mo-C steels with shape memory effect. Materials Science and Engineering: A, 2008, vol. 481-482, pp. 747-751. DOI: 10.1016/j.msea.2007.02.158.

5. Sagaradze V.V., Voronin V.I., Filippov Yu.I., Kazantsev V.A., Mukhin M.L., Belozerov E.V., Pecherkina N.L., Kataeva N.V., Popov A.G. Martensitic transformations $\gamma-\varepsilon(\alpha)$ and the shape-memory effect in aging high-strenght manganese austenitic steels. The Physics of Metals and Metallography, 2008, vol. 106, iss.6, pp 630-640. DOI: 10.1134/S0031918X08120120.

6. Sagaradze V. V., Afanas'ev S. V., Kataeva N. V. Shape memory effect in Mn-V-C austenitic steels involving deformation reorientation of $\varepsilon$-martensite. The Physics of Metals and Metallography, 2013, vol. 114, iss. 4, pp. 322-326. DOI: 10.1134/S0031918X13020117.

7. Gavriljuk V.G., Bliznuk V.V., Shanina V.D., Kolesnik S.P. Effect of silicon on atomic distribution and shape memory in Fe-Mn base alloys. Materials Science and Engineering: A, 2005, vol. 406, iss. 1-2, pp. 1-10. DOI: 10.1016/j.msea.2005.05.043.

8. Baruj A., Kikuchi T., Kajivara S., Shinya N. Improved shape memory properties and internal structures in Fe-Mn-Si-based alloys containing $\mathrm{Nb}$ and C. J. Phys. IV France, 2003, vol. 112, pp. 373-376. DOI: 10.1051/jp4:2003904. 
9. Dong Z.Z., Kajiwara S., Kikuchi T., Sawaguchi T. Effect of pre-deformation at room temperature on shape memory properties of stainless type Fe-15Mn-5Si-9Cr-5Ni-(0.5-1 .0) NbC alloys. Acta Materialia, 2005, vol. 53, iss. 15, pp. 4009-4018. DOI: 10.1016/j.actamat.2005.04.035.

10. Malyshev K.A., Vasilevskaya M. A change in the physical properties of martensite in titanium-alloyed iron-nickel alloys under tempering. Fizika Metallov i Metallovedenie, 1964, vol. 18, no. 5, pp. 793-795. (In Russian).

11. Sagaradze V.V., Uvarov A.I. Uprochnenie i svoistva austenitnykh stalei [Hardening and Properties of Austenitic Steels]. Ekaterinburg, RIO UO RAS Publ., 2013, 720 p. (In Russian).

12. Romanova R.R., Pushin V.G., Uksusnikov A.N., Buinov N.N. Strukturnyy mekhanizm stareniya austenitnykh zhelezomargantsevykh i zhelezonikelevykh stalei, uprochnyaemykh karbidom vanadiya. In: Struktura i svoistva nemagnitnykh stalei [Structure and Properties of Non-Magnetic Steels]. M., Nauka Publ., 1982, pp. 107-114.

13. Van Aswegen J.S.T., Honeycombe R.W.K. Segregation and precipitation in stacking faults. Acta Metallurgica, 1962, vol.10, iss. 3, pp. 262-264. 
Подана в журнал: 28.09.2015 г.

УДК 669.74'292'784:536.424.1

DOI: $10.17804 / 2410-9908.2015 .6 .019-026$

\title{
СТРУКТУРА И СВОЙСТВА УПРОЧНЕННЫХ НЕРЖАВЕЮЩИХ СТАЛЕЙ С ЭФФЕКТОМ ПАМЯТИ ФОРМЫ
}

\author{
С. В. Афанасьев, Е. Г. Волкова*, В. В. Сагарадзе \\ Федеральное государственное бюджетное учреждение науки Институт физики металлов имени \\ М.Н. Михеева Уральского отделения Российской академии наук, ул. С.Ковалевской, 18, 620990, \\ Екатеринбург, Российская Федераџия
}

\begin{abstract}
*Ответственный автор. Электронная почта: volkova@imp.uran.ru; адрес для переписки: ул. С. Ковалевской, 18, 620990, Екатеринбург, Российская Федерация. Телефон: +7 (343) 374-40-54; факс: +7 (343) 374-52-44
\end{abstract}

Исследована возможность получения эффекта памяти формы (ЭПФ) в результате $\gamma \rightarrow \varepsilon \rightarrow \gamma$ превращений в стареющих нержавеющих сталях, упрочняемых карбидами VC. Представлены режимы упрочняющего старения при 650 и $720^{\circ} \mathrm{C}$ нержавеющих сталей, преимущественно содержащих (мас. \%) 0,20-0,35 C, 1 V, 3-4 Si, 9 и 13-14 Cr. Значения обратимой деформации $e$ (величины ЭПФ), определенные после нагрева до $400{ }^{\circ} \mathrm{C}$ в предварительно деформированных на 3,5-4 \% образцах, изменяются от 0,15 до 2,7 \% в зависимости от состава сталей и режимов стабилизирующего и дестабилизирующего старения.

Ключевые слова: эффект памяти формы, стареюшие нержавеющие стали, карбид VC, мартенситное превращение, є-фаза, электронная микроскопия.

\section{1. Введение}

Дисперсионно-твердеющие Mn-Si-V стали с 0,2 - 1,0 мас. \% углерода (0,2C-20Mn-2Si-1V; 0,4C-18Mn-2Si-2V и др.), обладающие эффектом памяти формы (ЭПФ) до 2,5 \%, впервые были предложены и исследованы в работах [1-6]. Предложенные стали упрочняются за счет выделения наноразмерных карбидов VC при старении, испытывают прямое $\gamma \rightarrow \varepsilon$ и обратное $\varepsilon \rightarrow \gamma$ мартенситные превращения и имеют преимущества перед известными [7] ЭПФсталями на основе $\mathrm{Fe}-28 \mathrm{Mn}-6 \mathrm{Si}$. Они являются высокопрочными за счет возможности дисперсионного твердения, содержат существенно меньше марганца и кремния, позволяют регулировать величину ЭПФ в результате стабилизирующего или дестабилизирующего старения. Эффект дисперсионного твердения в этих ванадийсодержащих сталях с 0,2-1,0 мас. \% углерода выше, чем в ЭПФ-сталях с $0,5-1,0$ мас. \% ниобия [8, 9]. Однако предложенные [1-6] дисперсионно-твердеющие стали с эффектом памяти формы не являются коррозионностойкими. Представляет значительный интерес получить эффект памяти формы на стареющих нержавеющих сталях с повышенным содержанием хрома. В настоящее время нет ясности, как будет проходить старение в сталях с повышенным содержанием сильных карбидообразующих элементов - ванадия и хрома как изменятся механические свойства и величина ЭПФ в сталях с двумя видами карбидов разного размера, морфологии и расположения. Настоящая работа посвящена исследованию $\mathrm{Cr}-\mathrm{Mn}-\mathrm{V}-\mathrm{Si}$ аустенитных нержавеющих сталей с эффектом памяти формы.

\section{2. Материал и методика}

Исследованию подвергали дисперсионно-твердеющие аустенитные стали с эффектом памяти формы, имеющие разное содержание углерода, хрома, марганца, никеля, кремния и ванадия (таблица) и образующие при старении карбиды ванадия и хрома. Стали выплавляли в индукционной печи, разливали в слитки преимущественно массой 0,2 кг, проковывали и гомогенизировали при 1150-1200 ${ }^{\circ} \mathrm{C}$. Определение величины ЭПФ выполняли после старе- 
ния, прямого мартенситного $\gamma \rightarrow \varepsilon$ превращения при холодной деформации и обратного мартенситного превращения $\varepsilon \rightarrow \gamma$ в результате нагрева до $450-500{ }^{\circ} \mathrm{C}$. Старение осуществляли при 650-750 ${ }^{\circ} \mathrm{C}$ с выдержкой до 10 ч. После закалки от $1100-1150{ }^{\circ} \mathrm{C}$ и старения пластинчатые образцы подвергали холодной деформации изгибом на $180^{\circ}$ вокруг цилиндрических оправок диаметром 30 или 60 мм с целью осуществления мартенситного $\gamma$ - $\varepsilon$ превращения. Исходную деформацию $\left(e_{0}, \%\right)$ на внешней поверхности изогнутого пластинчатого образа рассчитывали по известной формуле $e_{\mathrm{o}}=100 \mathrm{~b} / D$, где $b$ - толщина пластины, а $D$ - диаметр изогнутого образца. При этом деформация растяжения на внешней поверхности изогнутого образца была равна 3,5-4 \%. Температурный интервал обратного $\varepsilon-\gamma$ превращения при нагреве определяли дилатометрически. В частности, в закаленной и деформированной на 3,5\% стали 0,20C-9Cr-15Mn-3Ni-4Si-1V $\varepsilon \rightarrow \gamma$ превращение происходит в интервале $80-320{ }^{\circ} \mathrm{C}$. Обратимую деформацию $e$ (величину ЭПФ, \%) рассчитывали по разнице между исходной холодной деформацией и оставшейся деформацией $\left(e_{\mathrm{r}}, \%\right)$ после распрямления образца в результате нагрева. Структуру и фазовый состав сталей анализировали методом рентгенографии и трансмиссионной электронной микроскопии в электронном микроскопе JEM-200CX.

\section{3. Результаты и обсуждение}

Изменение величины обратимой деформации от времени старения при $650{ }^{\circ} \mathrm{C}$ и $720{ }^{\circ} \mathrm{C}$ в сталях с 9 и 13-14 мас. \% хрома и 0,20-0,35 мас. \% углерода представлено в таблице. В дальнейшем для удобства будем указывать стали по номерам (таблица).

Увеличение времени «высокотемпературного» дестабилизирующего старения сталей с $14 \%$ Cr при $720^{\circ} \mathrm{C}$ приводит к прогрессирующему освобождению аустенитной матрицы от углерода и ванадия, что интенсифицирует образование $\varepsilon$-мартенсита деформации и способствует повышению величины ЭПФ в результате $\gamma \rightarrow \varepsilon \rightarrow \gamma$ превращения. Однако, в стали № 2 с повышенным содержанием углерода наблюдается перестаривание и охрупчивание образцов после выдержки в течение 6 ч при $720^{\circ} \mathrm{C}$. Сталь с меньшим содержанием хрома (9 мас. \%) и 0,2 мас. \% углерода в закаленном и состаренном (при $720{ }^{\circ} \mathrm{C}, 5-10$ ч) состояниях имеет высокие значения ЭПФ (e=2,4-2,7 \%) (таблица).

Величина эффекта памяти формы (e, \%) в нержавеющих сталях № 1-4 в зависимости от времени старения при $650{ }^{\circ} \mathrm{C}$ и $720{ }^{\circ} \mathrm{C}$.

\begin{tabular}{|c|c|c|c|}
\hline № & Сталь & Обработка & $e, \%$ \\
\hline \multirow[t]{5}{*}{1} & \multirow[t]{5}{*}{$0,20 \mathrm{C}-14 \mathrm{Cr}-15 \mathrm{Mn}-1 \mathrm{Ni}-4 \mathrm{Si}-1 \mathrm{~V}$} & Закалка от $1100^{\circ} \mathrm{C}$ & 1,0 \\
\hline & & $650^{\circ} \mathrm{C}, 3$ ч & 0,8 \\
\hline & & $650^{\circ} \mathrm{C}, 6$ ч & 0,9 \\
\hline & & $720^{\circ} \mathrm{C}, 3$ ч & 1,0 \\
\hline & & $720^{\circ} \mathrm{C}, 6$ ч & 1,4 \\
\hline \multirow[t]{3}{*}{2} & \multirow[t]{3}{*}{ 0,35-14Cr-15Mn-3Ni-4Si-1V } & Закалка от $1100^{\circ} \mathrm{C}$ & 0,2 \\
\hline & & $720^{\circ} \mathrm{C}, 1$ ч & 0,8 \\
\hline & & $720^{\circ} \mathrm{C}, 3$ ч & 1,3 \\
\hline \multirow[t]{4}{*}{3} & \multirow[t]{4}{*}{ 0,30C-13Cr-15Mn-3Si-1V } & Закалка от $1100^{\circ} \mathrm{C}$ & 0,5 \\
\hline & & $720^{\circ} \mathrm{C}, 1$ ч & 0,6 \\
\hline & & $720^{\circ} \mathrm{C}, 3$ ч & 0,5 \\
\hline & & $720^{\circ} \mathrm{C}, 6$ ч & 1,1 \\
\hline \multirow[t]{6}{*}{4} & \multirow[t]{6}{*}{ 0,20C-9Cr-15Mn-3Ni-4Si-1V } & Закалка от $1100^{\circ} \mathrm{C}$ & 2,4 \\
\hline & & $720^{\circ} \mathrm{C}, 1$ ч & 1,5 \\
\hline & & $720^{\circ} \mathrm{C}, 3$ ч & 1,8 \\
\hline & & $720^{\circ} \mathrm{C}, 5$ ч & 2,6 \\
\hline & & $720^{\circ} \mathrm{C}, 6$ ч & 2,7 \\
\hline & & $720^{\circ} \mathrm{C}, 10$ ч & 2,5 \\
\hline
\end{tabular}


open-recess journal

Сопоставим структуру и свойства ЭПФ-сталей с 9 (№ 4) и 13-14 (№ 1-3) мас. \% хрома (таблица) после старения, $\gamma \rightarrow \varepsilon$ превращения при деформации $\sim 4 \%$ и обратного $\varepsilon \rightarrow \gamma$ превращения при нагреве до $500{ }^{\circ} \mathrm{C}$. На примере стали № 1, с 14 мас. \% Cr, видно, что «низкотемпературное» старение $\left(650{ }^{\circ} \mathrm{C}, 3\right.$ и 6 ч) не приводит к увеличению эффекта памяти формы. Как известно $[2,10,11]$, в этом случае происходит частичная стабилизация аустенита по отношению к мартенситному $\gamma \rightarrow \varepsilon$ превращению (образуется меньше $\varepsilon$-мартенсита при исходной деформации и, как следствие, снижается величина обратимой деформации при нагреве). Такая стабилизация аустенита имеет место $[10,11]$ несмотря на обеднение аустенитной матрицы углеродом и ванадием в процессе старения при $650{ }^{\circ} \mathrm{C}$. Увеличение температуры старения до $720^{\circ} \mathrm{C}$ (6 ч) приводит к дестабилизации аустенита и росту ЭПФ до 1,4 \% (таблица).

В структуре сталей № 1-3, с 13-14 \% Сr, после старения при 720 (6 ч) и цикла $\gamma \rightarrow \varepsilon \rightarrow \gamma$ превращений (рис. $1 a-8$ ) сохраняются карбиды $\mathrm{VC}$, дефекты упаковки и вновь появляются отдельные редкие кристаллы $\varepsilon$-мартенсита. В результате подобной обработки в стали № 1 , с меньшим пересыщением по углероду, частицы VC (с размером до 12 нм и плотностью $7 \times 10^{14}$ $\mathrm{cm}^{-3}$ ) расположены преимущественно гетерогенно на дислокациях (рис. $1 a$ ), как и в работе [12]. В стали № 3, с 0,30 мас. \% C, карбидная фаза VC располагается достаточно равномерно по объему зерен, имеет средний размер $\sim 6$ нм и более высокую плотность $3 \times 10^{15} \mathrm{~cm}^{-3}$ (рис. 1 в). Кроме дисперсных карбидов $\mathrm{VC}$ в процессе старения при $720{ }^{\circ} \mathrm{C}$ в отдельных случаях появляются более крупные частицы карбида $\mathrm{Cr}_{23} \mathrm{C}_{6}$ (рис. 1 г), которые обычно ответственны за уменьшение пластичности и охрупчивание сталей. Таким образом, в сталях с повышенным содержанием хрома (13-14 мас. \%) старение не вызывает необходимую дестабилизацию аустенита (для получения наибольшего количества $\varepsilon$-мартенсита деформации), что определяет не очень высокие значения ЭПФ $(e=1,3-1,4 \%)$.

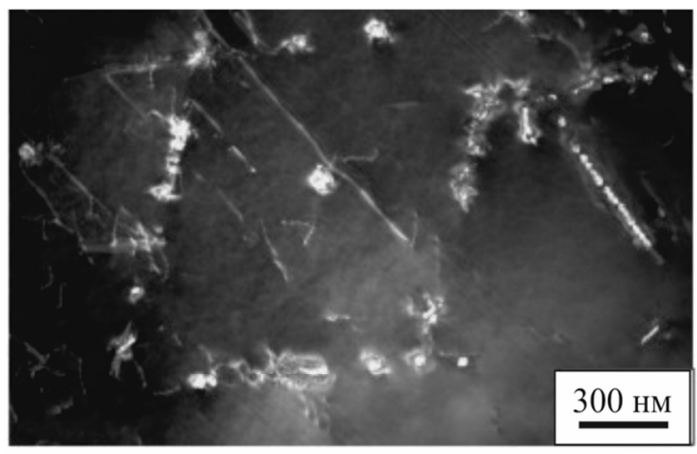

$a$

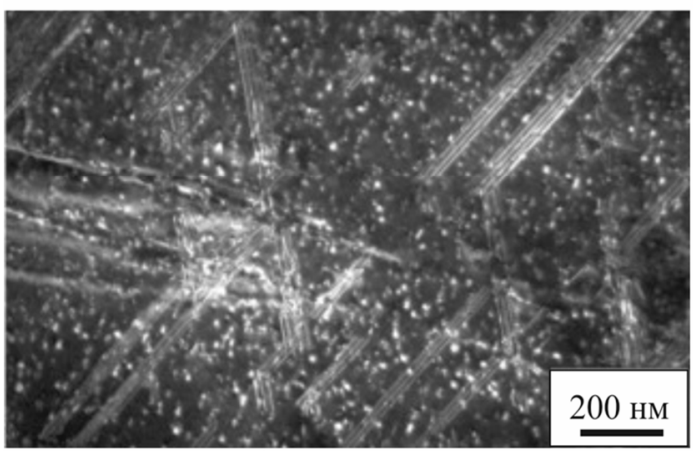

B

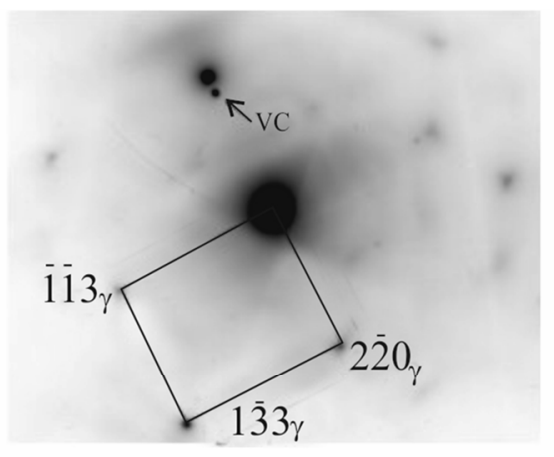

6

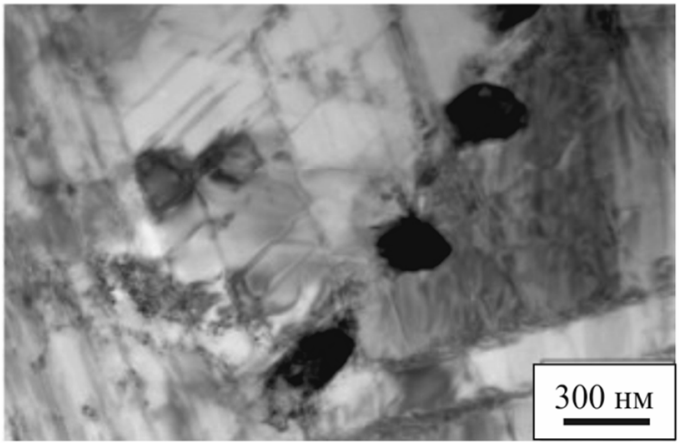

2

Рис. 1. Структура и электронограмма сталей № 1 ( $a$, б), № 3 (в), № 2 (2) с темнопольным изображением в рефлексе $\mathrm{VC}(a, b)$. Обработка: закалка от $1100{ }^{\circ} \mathrm{C}$; старение при $720{ }^{\circ} \mathrm{C}, 6$ ч; холодная деформация $\sim 4 \%$ ( $\gamma \rightarrow \varepsilon$ превращение) и нагрев до $500{ }^{\circ} \mathrm{C}(\varepsilon \rightarrow \gamma$ превращение) 
onen-reCESS journal

В стали № 4, с 9 мас. \% хрома и 0,2 мас. \% углерода, наблюдается максимальная величина обратимой деформации $(e=2,5-2,7 \%)$ после дестабилизирующего старения $\left(720{ }^{\circ} \mathrm{C}\right.$, 5-10 ч). Малое время старения (1-3 ч) при $720^{\circ} \mathrm{C}$ в этой стали приводит к некоторой стабилизации аустенита и уменьшает величину ЭПФ (e=1,5-1,8 \%). На рентгенограмме (рис. 2) представлены отражения от $\gamma$ и $\varepsilon$ фаз в стали № $4-0,20 \mathrm{C}-9 \mathrm{Cr}-15 \mathrm{Mn}-3 \mathrm{Ni}-4 \mathrm{Si}-1 \mathrm{~V}$ после старения при $720{ }^{\circ} \mathrm{C}(3$ ч) и деформации изгибом на $\sim 4 \%$. Количество $\varepsilon$-мартенсита после такой обработки составляет $\sim 40 \%$, что больше, чем во всех исследованных деформированных образцах сталей с 13-14 мас. \% хрома.

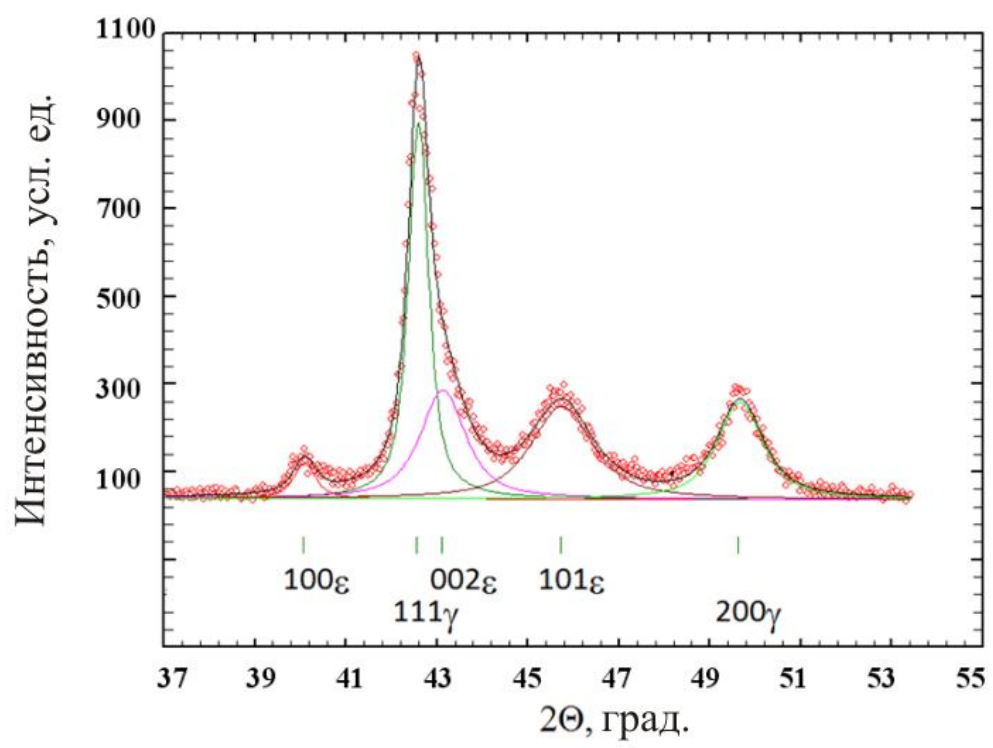

Рис. 2. Рентгенограмма стали № 4 с отражениями от $\varepsilon$ - и $\gamma$-фаз. Обработка: закалка от 1100 ${ }^{\circ} \mathrm{C}$, старение при $720{ }^{\circ} \mathrm{C}$ (3 ч), холодная деформация на 3,5 \% изгибом

На рис. $3 a$, $б$ показана структура стали после предварительной закалки и старения $\left(720^{\circ} \mathrm{C}, 1\right.$ ч). Видны частицы VC размером $\sim 10$ нм, равномерно распределенные в матрице (плотность $5 \times 10^{14} \mathrm{~cm}^{-3}$ ). Присутствуют множественные дефекты упаковки. Вокруг частиц хорошо заметны поля напряжений, создающие контраст в виде крыльев бабочки (рис. $3 a$ ). При увеличении времени старения до 3 ч структура существенно не меняется. Отдельные частицы VC зарождаются на дислокациях при старении и становятся источниками расщепления полных дислокаций на частичные с образованием дефектов упаковки. На образующихся дефектах упаковки формируются новые мелкие частицы, которые своим упругим полем способствуют дальнейшему расщеплению дислокаций. На рис. 3 в, г в состаренной при $720{ }^{\circ} \mathrm{C}$, 6 ч, стали № 4 можно видеть дефекты упаковки, на которых выделились частицы VC (средний размер 9 нм, плотность $\left.3 \times 10^{15} \mathrm{~cm}^{-3}\right)$. Аналогичная ситуация наблюдалась в работе [13], в которой на аустенитной стали $0,1 \mathrm{C}-18 \mathrm{Cr}-10 \mathrm{Ni}-1 \mathrm{Nb}$ впервые было показано, что старение при $700{ }^{\circ} \mathrm{C}$ (5 ч) снижает энергию дефектов упаковки и приводит к диссоциации полных дислокаций с образованием дефектов упаковки. Увеличение времени старения при $700{ }^{\circ} \mathrm{C}$ также вызвало формирование дисперсных карбидов (NbC) на дефектах упаковки [13]. 
Open-acCeEs journal

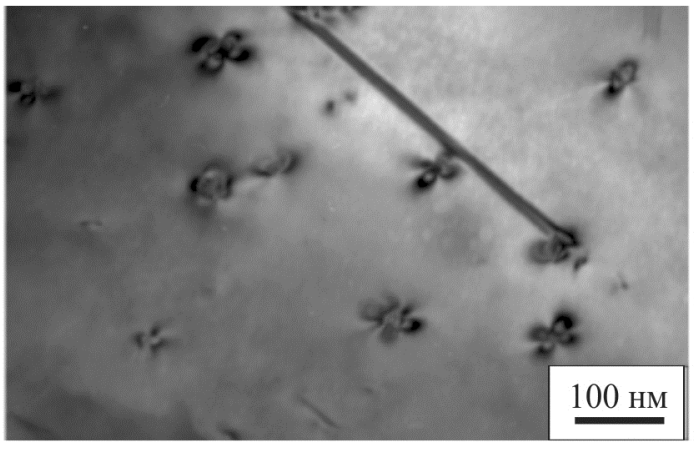

$a$

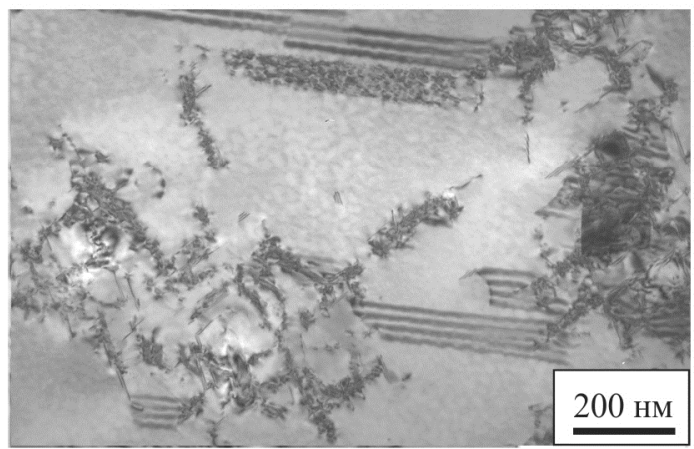

B

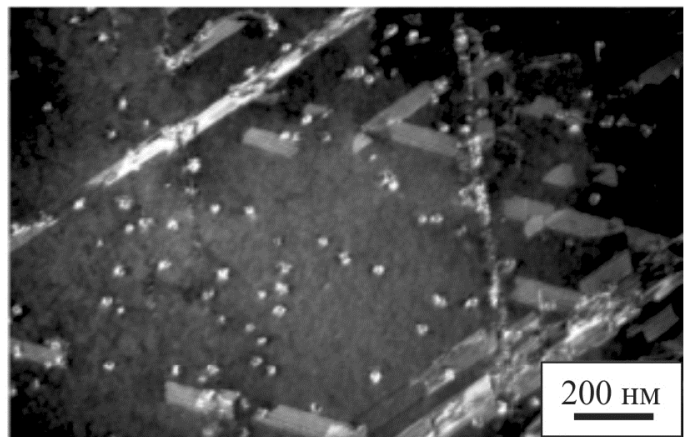

б

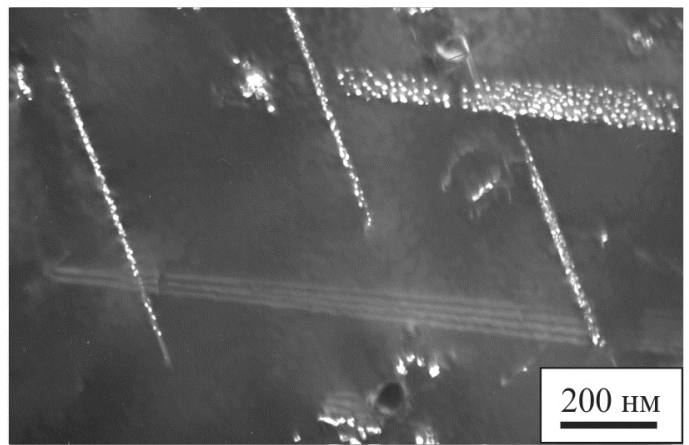

2

Рис. 3. Светлопольные $(a, b)$ и темнопольные - в составном рефлексе $\gamma+\mathrm{VC}(\sigma, 2)$ изображения структуры стали № 4. Обработка: закалка от $1100{ }^{\circ} \mathrm{C}$, старение при $720^{\circ} \mathrm{C}, 1$ ч $(a, \sigma)$, или $720{ }^{\circ} \mathrm{C}, 6$ ч $(в, 2)$, с последующим $\gamma \rightarrow \varepsilon \rightarrow \gamma$ превращением

Таким образом, увеличение времени старения стали № 4 до 6 ч при $720{ }^{\circ} \mathrm{C}$ способствует смене механизма зарождения карбидов с гомогенного на гетерогенный, что приводит к увеличению ЭПФ $(e=2,7 \%)$.

\section{4. Заключение}

1. Показано, что наибольшая величина обратимой деформации наблюдается в коррозионно-стойких ЭПФ-сталях с 9 мас. \% хрома, 0,20 мас. \% углерода и высоким содержанием кремния (4 мас. \%), который уменьшает энергию дефекта упаковки, способствует образованию $\varepsilon$-фазы с ГПУ-решеткой и увеличивает ЭПФ $(e=2,50-2,70$ \%).

2. В исследованных коррозионно-стойких ЭПФ-сталях с 13-14 мас. \% хрома наблюдаются существенно меньшие значения обратимой деформации (преимущественно $e=1,0-1,4 \%$ ), чем в сплаве с 9 мас. \% хрома, даже после дестабилизирующего старения при $720{ }^{\circ} \mathrm{C}\left(6\right.$ ч) с выделением карбидов $\mathrm{VC}$ и $\mathrm{Cr}_{23} \mathrm{C}_{6}$.

3. Смена механизма выделения карбидов с гомогенного на гетерогенный при повышении температуры и времени старения способствует росту величины обратимой деформации в нержавеющих ЭПФ-сталях.

\section{Благодарность}

Электронно-микроскопические исследования проведены в ОЭМ ЦКП ИФМ УрО РАН. Работа выполнена в рамках государственного задания ФАНО России (тема «Структура», № 01201463331) и при частичной поддержке Уральского отделения РАН (проект № 15-9-2-16). 


\section{Литература}

1. The shape memory effect in high-strength precipitation-hardening austenitic steels / V. V. Sagaradze, Ye. V. Belozerov, N. L. Pecherkina, M. L. Mukhin, Yu. R. Zaynutdinov // Materials Science and Engineering: A. - 2006. - Vol. 438-440. - P. 812-815. - DOI:10.1016/j.msea.2006.01.113.

2. A new approach to creation of high-strength austenitic steels with a controlled shapememory effect / V. V. Sagaradze, E. V. Belozerov, M. L. Mukhin, Yu. R. Zainutdinov, N. L. Pecherkina, V. A. Zavalishin // The Physics of Metals and Metallography. - 2006. - Vol. 101, iss. 5. P. 506-512. - DOI: 10.1134/S0031918X06050127.

3. Controlled shape memory effect in high strength $\mathrm{Mn}$ and Cr-Mn steels / V. V. Sagaradze, M. L. Mukhin, E. V. Belozerov, Yu. R. Zainutdinov, N. L. Pecherkina, Yu. I. Filippov//Materials Science and Engineering: A. - 2008. - Vol. 481-482. - P. 742-746. - DOI: 10.1016/j.msea.2007.02.155.

4. High-strength precipitation-hardening austenitic Fe-Mn-V-Mo-C steels with shape memory effect / V. V. Sagaradze, I. I. Kositsyna, M. L. Mukhin, E. V. Belozerov, Yu. R. Zaynutdinov // Materials Science and Engineering: A. - 2008. - Vol. 481-482. - P. 747-751. - DOI: 10.1016/j.msea.2007.02.158.

5. Martensitic transformations $\gamma-\varepsilon(\alpha)$ and the shape-memory effect in aging high-strenght manganese austenitic steels / V. V. Sagaradze, V. I. Voronin, Yu. I. Filippov, V. A. Kazantsev, M. L. Mukhin, E. V. Belozerov, N. L. Pecherkina, N. V. Kataeva, A. G. Popov // The Physics of Metals and Metallography. - 2008. -Vol. 106, iss.6. - P. 630-640. - DOI: 10.1134/S0031918X08120120.

6. Sagaradze V. V., Afanas'ev S. V., Kataeva N. V. Shape memory effect in Mn-V-C austenitic steels involving deformation reorientation of $\varepsilon$-martensite // The Physics of Metals and Metallography. - 2013. - Vol. 114, iss. 4. - P. 322-326. - DOI: 10.1134/S0031918X13020117.

7. Effect of silicon on atomic distribution and shape memory in Fe-Mn base alloys / V. G. Gavriljuk, V. V. Bliznuk, V. D. Shanina, S. P. Kolesnik // Materials Science and Engineering: A. -2005 . - Vol. 406, iss. 1-2. - P. 1-10. - DOI: 10.1016/j.msea.2005.05.043.

8. Improved shape memory properties and internal structures in Fe-Mn-Si-based alloys containing $\mathrm{Nb}$ and C / A. Baruj, T. Kikuchi, S. Kajivara, N. Shinya // J. Phys. IV France. - 2003. - Vol. 112. P. 373-376. - DOI: 10.1051/jp4:2003904.

9. Effect of pre-deformation at room temperature on shape memory properties of stainless type Fe-15Mn-5Si-9Cr-5Ni-(0.5-1.0)NbC alloys / Z. Z. Dong, S. Kajiwara, T. Kikuchi, T. Sawaguchi // Acta Materialia. - 2005. - Vol. 53, iss. 15. - P. 4009-4018. - DOI: 10.1016/j.actamat.2005.04.035.

10. Малышев К. А., Василевская М. Изменение физических свойств при отпуске мартенсита в железоникелевых сплавах, легированных титаном // ФММ. - 1964. - Т. 18, № 5. С. 793-795.

11. Сагарадзе В. В., Уваров А. И. Упрочнение и свойства аустенитных сталей. - Екатеринбург : РИО УрО РАН, 2013. - 720 с.

12. Структурный механизм старения аустенитных железомарганцевых и железоникелевых сталей, упрочняемых карбидом ванадия / Р. Р. Романова, В. Г. Пушин, А. Н. Уксусников, Н. Н. Буйнов // Структура и свойства немагнитных сталей. - М. : Наука, 1982. - С. 107-114.

13. Van Aswegen J. S. T., Honeycombe R. W. K. Segregation and precipitation in stacking faults // Acta Metallurgica. -1962. - Vol.10, iss. 3. - P. 262-264. 